\title{
Inelastic current-voltage characteristics of atomic and molecular junctions
}

\author{
Yu-Chang Chen \\ Department of Physics, University of California, San Diego, La Jolla, CA 92093-0319 \\ Michael Zwolak \\ Department of Physics, California Institue of Technology, Pasadena, California 91125 \\ Massimiliano Di Ventra* \\ Department of Physics, University of California, San Diego, La Jolla, CA 92093-0319
}

\begin{abstract}
We report first-principles calculations of the inelastic current-voltage (I-V) characteristics of a gold point contact and a molecular junction in the nonresonant regime. Discontinuities in the I- $\mathrm{V}$ curves appear in correspondence to the normal modes of the structures. Due to the quasi-onedimensional nature of these systems, specific modes with large longitudinal component dominate the inelastic I-V curves. In the case of the gold point contact, our results are in good agreement with recent experimental data. For the molecular junction, we find that the inelastic I-V curves are quite sensitive to the structure of the contact between the molecule and the electrodes thus providing a powerful tool to extract the bonding geometry in molecular wires.
\end{abstract}

Inelastic scattering between electrons and phonons in a current-carrying wire is a source of energy dissipation for electrons. However, it can also yield a lot of information on the underlying atomic structure of the wire. This information can be extracted indirectly from the discontinuities in conductance that occur when the external bias is large enough to excite discrete vibrational modes of the wire. 1 Recent experiments on transport properties of atomic 2] and molecular [3, 4] junctions have indeed revealed such inelastic features. It is, however, not straightforward to relate these features to specific vibrational modes. A nanoscale junction (often described as a quasione-dimensional system) with $N$ atoms supports $3 N$ vibrational modes. In a strictly one-dimensional system, only longitudinal modes can be excited via electronic coupling. However, the modes of a realistic junction are not necessarily purely transverse or purely longitudinal with respect to the direction of current flow. [5, [6, 7] Therefore, the inelastic current-voltage (I-V) characteristics are likely to depend strongly on the detailed atomic structure of the full system. This is particularly relevant for molecular junctions for which the contact geometry between the molecule and the bulk electrodes is difficult to control in experiments. [8, [9]

In this letter we first derive an expression for the inelastic current in a current-carrying system in terms of scattering wavefunctions. This expression allows us to study the inelastic I-V characteristics of a given nanoscale junction using first-principles approaches. As an example we study the effect of vibrations on the electron dynamics in a gold point contact and a single-molecule junction. For the gold point contact, the magnitude of the calculated inelastic current as well as its onset compare very well with recent experimental results. 2] For the molecular junction, we analyze the case in which the molecule is equally bonded to the two bulk electrodes and the case in which the two contacts are different. We find that the inelastic I-V characteristics are very different in the two cases. This result shows that inelastic spectroscopy could be used quite effectively to extract information on the contact geometry of molecular wires.

Let us start by deriving an expression for the inelastic current. We assume that the phonon distribution is at equilibrium at all (small) biases, thus neglecting local heating. [5] We have previously shown that for small biases this effect is small, provided good thermal contacts exist between the nanostructures and the bulk electrodes. [5] The many-body Hamiltonian of the system is (atomic units are used throughout this paper) [5]

$$
H=H_{e l}+H_{v i b}+H_{e l-v i b},
$$

where $H_{e l}$ is the electronic part of the Hamiltonian; $H_{v i b}=\frac{1}{2} \sum_{i, \mu \in v i b} \dot{q}_{i \mu}^{2}+\frac{1}{2} \sum_{i, \mu \in v i b} \omega_{i \mu}^{2} q_{i \mu}^{2}$ is the ionic contribution where $q_{i \mu}$ is the normal coordinate and $\omega_{i \mu}$ is the normal frequency corresponding to the $i$-th ion and $\mu$ th component; finally, $H_{e l-v i b}$ describes the electron-ion interaction and has the following form:

$$
\begin{aligned}
H_{e l-v i b} & =\sum_{\alpha, \beta} \sum_{E_{1}, E_{2}} \sum_{i \mu, j \nu \in v i b} \\
& \sqrt{\frac{\hbar}{2 \omega_{j \nu}}} A_{i \mu, j \nu} J_{E_{1}, E_{2}}^{i \mu \beta} a_{E_{1}}^{\alpha \dagger} a_{E_{2}}^{\beta}\left(b_{j \nu}+b_{j \nu}^{\dagger}\right),
\end{aligned}
$$

where $\alpha=L, R ; a_{E}^{\alpha}$ and $b_{j \nu}$ are the electron and phonon annihilation operators, respectively, satisfying the usual commutation relations. $A_{i \mu, j \nu}$ are the matrix elements of 
the transformation from cartesian coordinates to normal coordinates, and $J_{E_{1}, E_{2}}^{i \mu, \beta}$ is the electron-phonon coupling constant which can be directly calculated from the scattering wave-functions [5]

$J_{E_{1}, E_{2}}^{i \mu, \alpha \beta}=\int d \mathbf{r} \int d \mathbf{K}_{\|} \Psi_{E_{1}}^{\alpha *}\left(\mathbf{r}, \mathbf{K}_{\|}\right) \partial_{\mu} V^{p s}\left(\mathbf{r}, \mathbf{R}_{i}\right) \Psi_{E_{2}}^{\beta}\left(\mathbf{r}, \mathbf{K}_{\|}\right)$,

where we have chosen to describe the electron-ion interaction with pseudopotentials $V^{p s}\left(\mathbf{r}, \mathbf{R}_{i}\right)$ for each i-th ion. [10]

Similar to what has been done in Ref. 6, we treat the electron-phonon interaction to first-order perturbation theory. 11] Due to the orthogonality condition between phonon states, higher harmonics for each phonon mode appear only in third-order perturbation theory and are therefore small. 12. We develop the full manybody wavefunctions in terms of the states $\left\langle\Psi_{E}^{L(R)} ; n_{j \nu}\right|=$ $\left\langle\Psi_{E}^{L(R)}\right| \otimes\left\langle n_{j \nu}\right|$. The single-particle electronic state is described by $\Psi_{E}^{L(R)}\left(\mathbf{r}, \mathbf{K}_{\|}\right)$, corresponding to electrons incident from the left (right) electrodes with energy $\mathrm{E}$ and momentum $\mathbf{K}_{\|}$parallel to the electrode surface. [10] These electronic states are calculated self-consistently by means of a scattering approach within the density functional theory of many-electron systems. 10] The phonon state is described by $\left\langle n_{j \nu}\right|$, where $n_{j \nu}$ is the number of phonons in the $j \nu$-th normal mode.

$$
\begin{aligned}
\left|\delta \Psi_{E}^{\alpha} ; n_{j \nu}\right\rangle & =\lim _{\epsilon \rightarrow 0^{+}} \sum_{\alpha^{\prime}=L, R} \sum_{j^{\prime} \nu^{\prime}} \int d E^{\prime} D_{E^{\prime}}^{\alpha^{\prime}} \\
& \frac{\left\langle\Psi_{E^{\prime}}^{\alpha^{\prime}} ; n_{j^{\prime} \nu^{\prime}}\left|H_{e l-v i b}\right| \Psi_{E}^{\alpha} ; n_{j \nu}\right\rangle\left|\Psi_{E^{\prime}}^{\alpha^{\prime}} ; n_{j^{\prime} \nu^{\prime}}\right\rangle}{\varepsilon\left(E, n_{j \nu}\right)-\varepsilon\left(E^{\prime}, n_{j^{\prime} \nu^{\prime}}\right)-i \epsilon} .
\end{aligned}
$$

In the above expression $D_{E}^{\alpha}$ is the partial density of states corresponding to the current-carrying states $\Psi_{E}^{\alpha}$ and $\varepsilon\left(E, n_{j \nu}\right)=E+\left(n_{j \nu}+1 / 2\right) \hbar \omega_{j \nu}$ is the energy of state $\left|\Psi_{E}^{\alpha} ; n_{j \nu}\right\rangle$. We have also assumed that the electrons rapidly thermalize into the bulk electrodes so that their statistics are given by the equilibrium Fermi-Dirac distribution, $f_{E}^{L(R)}=1 /\left(\exp \left[\left(E-E_{F L(R)}\right) / k_{B} T_{e}\right]+1\right)$ with chemical potential $E_{F L(R)}$ deep into the left (right) electrode. 13] Using $\lim _{\epsilon \rightarrow 0} \frac{1}{z-i \epsilon}=P\left(\frac{1}{z}\right)+i \pi \delta(z)$, the firstorder correction $\left|\delta \Psi_{E}^{\alpha} ; n_{j \nu}\right\rangle$ assumes the following form:

$$
\begin{gathered}
\left|\delta \Psi_{E}^{R} ; n_{j \nu}\right\rangle=i \pi \sum_{i \mu \in v i b} \sqrt{\frac{\hbar}{2 \omega_{j \nu}}} A_{i \mu, j \nu} \\
{\left[D_{E+\hbar \omega_{j \nu}}^{L} \sqrt{\left\langle n_{j \nu}\right\rangle f_{E}^{R}\left(1-f_{E+\hbar \omega_{j \nu}}^{L}\right)} .\right.} \\
J_{E+\hbar \omega_{j \nu}, E}^{i \mu, L R}\left|\Psi_{E+\hbar \omega_{j \nu}}^{L} ; n_{j \nu}-1\right\rangle+ \\
D_{E-\hbar \omega_{j \nu}}^{L} \sqrt{\left(1+\left\langle n_{j \nu}\right\rangle\right) f_{E}^{R}\left(1-f_{E-\hbar \omega_{j \nu}}^{L}\right)} . \\
\left.J_{E-\hbar \omega_{j \nu}, E}^{i \mu, L R}\left|\Psi_{E-\hbar \omega_{j \nu}}^{L} ; n_{j \nu}+1\right\rangle\right],
\end{gathered}
$$

where $\left\langle n_{j \nu}\right\rangle=1 /\left[\exp \left(\hbar \omega_{j \nu} / k_{B} T_{w}\right)-1\right]$ is the BoseEinstein distribution per mode at a given wire temperature $T_{w}$, and \langle\rangle indicates the statistical average. The above expression allows us to calculate the inelastic current. It is evident from Eq. 5 that for a fixed partial density of states, the magnitude of the inelastic current is determined by the coupling constant $J_{E_{1}, E_{2}}^{i \mu, \alpha \beta}$ and the transformation matrix $\mathbf{A}=\left\{A_{i \mu, j \nu}\right\}$ which contains the information on the geometry of the structure and hence on the character (transverse versus longitudinal) of the different modes. 14 We will be concerned with the $e x$ tra inelastic current due to the vibrational modes of the atoms of the nanoscale constriction with respect to the continuum spectrum of modes of the bulk electrodes. 15] If the electronic temperature $T_{e}$ is zero, then, for an external bias $V$, only those normal modes with eigenenergies $\hbar \omega_{j \nu}<e V$ can be excited and contribute to Eq. [5 In addition, due to our assumption of negligible local heating, the averaged number of phonons $\left\langle n_{j \nu}\right\rangle$ is zero for all normal modes. In this case the first-order correction to the current induced by electron-phonon interaction assumes the following simple form:

$$
\begin{aligned}
& \delta I=-i \int_{E_{F L}}^{E_{F R}} d E \int d \mathbf{R} \int d \mathbf{K}_{\|} \\
& \left.\left[\left(\delta \Psi_{E}^{R}\right)^{*} \partial_{z} \delta \Psi_{E}^{R}-\partial_{z}\left(\delta \Psi_{E}^{R}\right)^{*} \delta \Psi_{E}^{R}\right)\right],
\end{aligned}
$$

where only the left-travelling electronic states contribute (if the left electrode is positively biased).

We are now ready to use the above expression to study inelastic scattering in specific systems. We choose to first study a gold point contact for which experimental results are available [2] and then discuss the case of a molecular junction. In Fig. 1 we plot the inelastic conductance for a single gold atom.

In the absence of inelastic scattering and for the bias

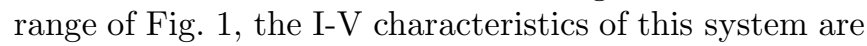
linear with differential conductance $G \simeq 1.1 G_{0}$, where $G_{0}=2 e^{2} / h$. [5] When electron-phonon interactions are considered, two transverse modes with energy $\hbar \omega \simeq 10.8$ $\mathrm{meV}$ are first excited with increasing bias. However, due to their transverse character, these modes contribute negligibly to the inelastic current as determined by the 


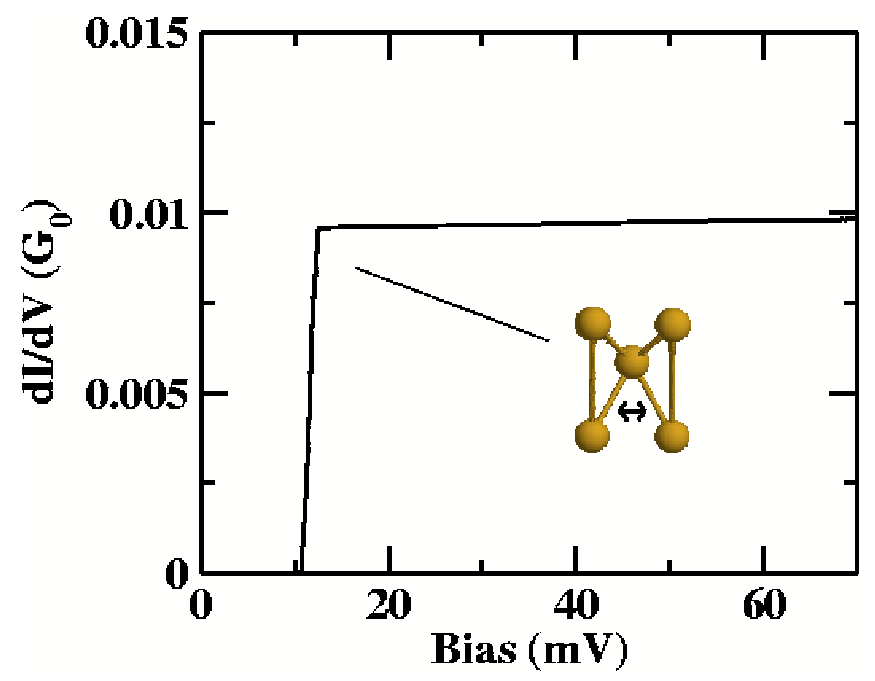

FIG. 1: Absolute value of the differential conductance due to electron-phonon interaction as a function of bias for a gold point contact. Two normal modes corresponding to transverse vibration of the gold atom between the electrodes have energies of $10.8 \mathrm{meV}$, which are close in energy to the longitudinal mode at $11.5 \mathrm{meV}$ (shown in the figure).

product between the transformation matrix $\mathbf{A}$ and the coupling constant $J_{E_{1}, E_{2}}^{i \mu \beta}$. An abrupt change in differential conductance appears at $V \simeq 11.5 \mathrm{mV}$ corresponding to the excitation of a longitudinal vibrational mode (see schematic in Fig. 11. 16] Both the onset bias as well as the change in conductance (of about $1 \%$ ) are in good agreement with experimental reports on gold point contacts. 2] The longitudinal and transverse modes are very close in energy but mainly the longitudinal mode contributes to the inelastic current, [6, 7] so that in experiments the transverse ones would not be easily resolved. This is even more evident in the case of the molecular junction.

In Fig. 2 we plot the inelastic conductance in the case in which a phenyldithiolate molecule forms symmetric contacts on both sides of the junction, i.e., each $\mathrm{S}$ atom is bonded to a flat surface. In this case there is a total of 14 modes with energy less than $100 \mathrm{meV}$. A prominent change in conductance occurs at a bias of about 18 $\mathrm{mV}$, i.e., at a bias large enough to excite two modes with large longitudinal component (see Fig. 22). 17] The inelastic contribution from two transverse modes at lower bias [5] is almost four orders of magnitude smaller. Similarly, three quasi-transverse modes with energies between 20 and $50 \mathrm{meV}$ contribute negligibly to the inelastic conductance. They only appear as small features in the second derivative of the current with respect to the bias (see Fig. 21). 18] It is likely that due to noise and other effects, such modes would not be resolved in experiments. Increasing the bias further, a second large step in the absolute value of the conductance is found at about 50 $\mathrm{mV}$ (see Fig. 2). This again corresponds to a predomi-

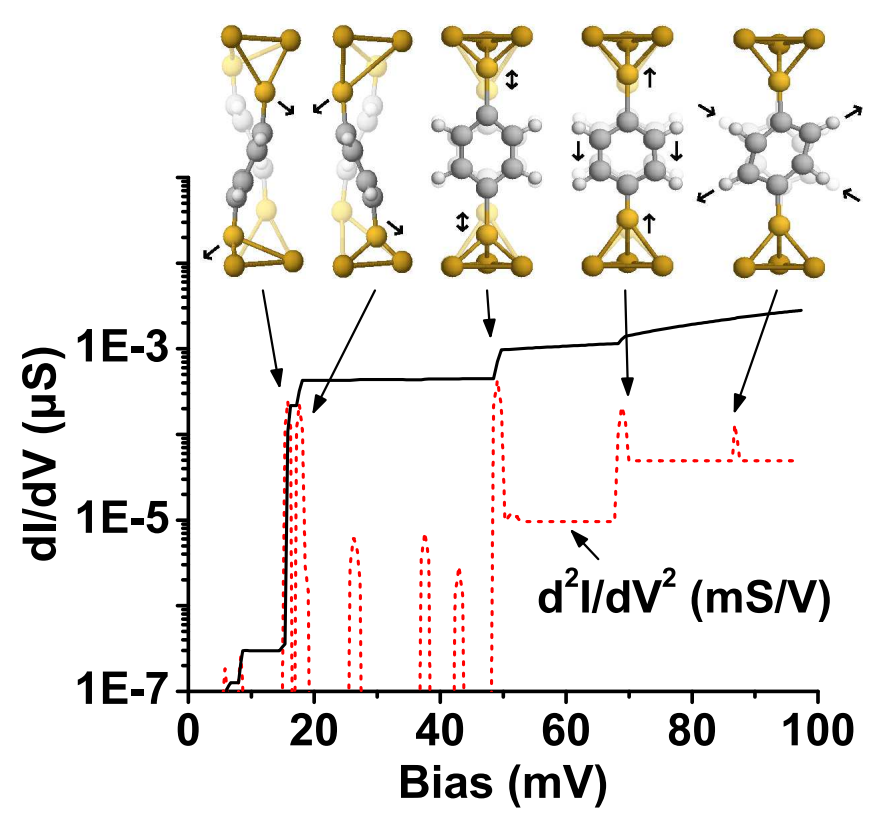

FIG. 2: Absolute value of the differential conductance due to electron-phonon interaction as a function of bias for a symmetric molecular junction. The derivative of the conductance with respect to bias is also shown (a broadening of $1 \mathrm{meV}$ is introduced). The schematics show only the modes that contribute the most to the inelastic current.

nantly longitudinal mode (see schematic in Fig. 2). This mode is then followed by others that have both a transverse and a longitudinal component. The magnitude of the conductance steps depends on the relative amount of the two components as well as the product between the transformation matrix $\mathbf{A}$ and the coupling constant. 17.

We conclude by showing how sensitive the inelastic current is to any change in the bonding properties of the molecule to the electrodes. We illustrate this in Fig. 3 where we plot the inelastic conductance for the same molecule but with one of the $\mathrm{S}$ atoms bonded to a $\mathrm{H}$ atom which, in turn, is not bonded to the nearby surface (see schematics in Fig. 3). 19] Such a configuration could be easily realized in experiments. [8] In the present case there are 13 modes below $100 \mathrm{meV}$, of which only six have large longitudinal component (shown in Fig. 31 17] and contribute to small steps in the inelastic conductance, with the one at about $11 \mathrm{meV}$ showing the largest relative contribution. All other modes contribute negligibly to the current. Comparing Figs. 2] and [3 it is clear that contact geometry affects considerably the I-V characteristics of the system both in the position of the inelastic discontinuities as well as in their relative magnitude. This fact, however, can be used advantageously to extract a posteriori the contact structure of molecular junctions thus providing a powerful diagnostic tool for nanoscale electronics.

We acknowledge support from the NSF Grant Nos. DMR-01-02277 and DMR-01-33075, and Carilion 


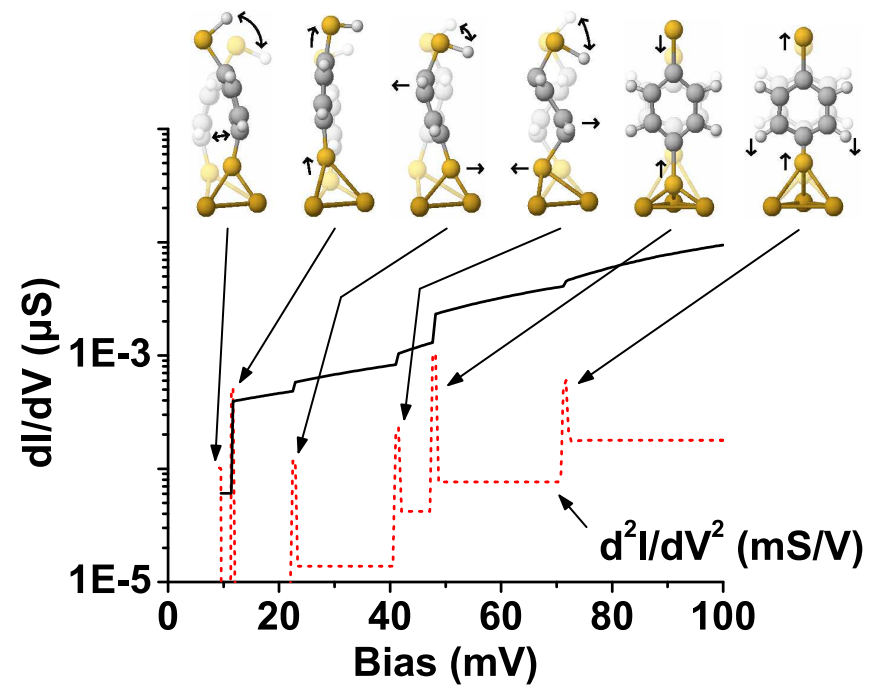

FIG. 3: Absolute value of the differential conductance due to electron-phonon interaction as a function of bias for a molecular junction with asymmetric contacts. The derivative of the conductance with respect to bias is also shown (a broadening of $1 \mathrm{meV}$ is introduced). The schematics show only the modes that contribute the most to the inelastic current.

Biomedical Institute. Acknowledgment is also made to the Donors of The Petroleum Research Fund, administered by the American Chemical Society, for partial support of this research. One of us (MZ) acknowledges partial support from an NSF Graduate Fellowship.

* E-mail address: diventra@physics.ucsd.edu

1 N. Lorente, M. Persson, L.J. Lauhon, W. Ho, Phys. Rev. Lett. 86, 2593 (2001).

2 N. Agraït, C. Untiedt, G. Rubio-Bollinger, and S. Vieira, Phys. Rev. Lett. 88, 216803 (2002).

${ }^{3}$ H. Park et al. Nature, 407,57 (2000); L.H. Yu and D. Natelson, Nano Letters, 4, 79 (2004).

${ }^{4}$ R.H.M. Smit, Y. Noat, C. Untiedt, N.D. Lang, M.C. van Hemert, and J.M. van Ruitenbeek, Nature 419, 906 (2002).

5 Y.-C. Chen, M. Zwolak, and M. Di Ventra, Nano Lett. 3, 1691 (2003).

6 M.J. Montgomery, J. Hoekstra, T.N. Todorov, and A. Sutton, J. Phys.: Cond. Mat. 15, 731 (2003).

7 A. Troisi, M.A. Ratner, and A. Nitzan, J. Chem. Phys. 118, 6072 (2003).

8 X. Xiao, B. Xu, and N.J. Tao, Nano Lett. 4, 267 (2004).

9 M. Di Ventra, S.T. Pantelides, and N.D. Lang, Phys. Rev. Lett. 84, 979 (2000).
10 N.D. Lang, Phys. Rev. B 52, 5335 (1995); M. Di Ventra and N.D. Lang, Phys. Rev. B 65, 045402 (2002); Z. Yang, A. Tackett, and M. Di Ventra, Phys. Rev B. 66, 041405 (2002).

11 Since we are in the regime of weak electron-phonon coupling we neglect the elastic phonon renormalization of the electronic spectrum (see e.g. G.D. Mahan, Many Particle Physics, 2nd Edition (Plenum Publishers, NY, 1990), p. 285).

12 V. Aji, J.E. Moore, and C.M. Varma, cond-mat/0302222 A. Mitra, I. Aleiner, A.J. Millis, cond-mat/0311503

13 Y.-C. Chen and M. Di Ventra, Phys. Rev. B 67,153304 (2003).

14 A quantitative way of distinguishing between longitudinal versus transverse character of each mode is to sum up the absolute values of all the $z$-component (i.e., along the current flow) matrix elements of $\mathbf{A}$.

15 We have employed Hartree-Fock total energy calculations [see, e.g., J. A. Boatz and M. S. Gordon, J.Phys. Chem., 93, 1819 (1989)] to evaluate the vibrational modes of the single-molecule junctions and the gold point contact. For these calculations, the assumed surface orientation is [111] represented by a triangular pad of gold atoms with infinite mass (see insets of Figs. [1 and 2). The structures were relaxed starting from an initial geometry where the single gold atom of the point contact faces the center of the triangular pad at a distance of $2.3 \AA$; and for the single-molecule junction with symmetric contacts the initial S-surface distance is $2.4 \AA$. In the case of asymmetric contacts the $\mathrm{S}$ atom on one side of the junction is bonded to a $\mathrm{H}$ atom (see insets of Fig. 3). This thiol termination is assumed to be far from the nearby surface so that the modes of this structure are not influenced by the gold atoms of the surface.

16 Note that the inelastic correction to the current is actually negative. For convenience we plot in this paper the absolute value of this correction.

17 In the molecular junction cases, the dominant net contribution to the electron-phonon coupling is from the $\mathrm{S}$ atoms and the adjacent $\mathrm{C}$ atoms (i.e., the two $\mathrm{C}$ atoms that form a straight line with the $\mathrm{S}$ atoms). This implies that those modes that do not have large displacements of these atoms along the $z$ direction will contribute negligibly to the inelastic current, even if the global character of the mode is longitudinal as inferred from the procedure outlined in Ref. 14. This is, e.g. the case for the mode at about 90 meV shown schematically in Fig. 2]

$18 \mathrm{~A}$ broadening of $1 \mathrm{meV}$ is introduced in all calculations to make the second derivative of the inelastic current as a function of bias finite at the conduction jumps.

19 In order to facilitate the comparison with the previous case, we have assumed in this calculation the same coupling constants, partial density of states and unperturbed current as for the symmetric junction case. 